Faraday Discussions

\title{
Spiers Memorial Lecture: Quantum Dynamics of Chemical Reactions
}

\author{
David C. Clary \\ Physical and Theoretical Chemistry Laboratory, \\ Department of Chemistry, \\ University of Oxford, \\ South Parks Road, \\ Oxford, OX1 3QZ, \\ United Kingdom. \\ Email: david.clary@chem.ox.ac.uk
}

\begin{abstract}
This Spiers Memorial Lecture discusses quantum effects that can be calculated and observed in the chemical reactions of small molecules. This includes quantum reactive scattering resonances, vibrational and rotational state effects, and quantum tunnelling in chemical reactions. Both experimental and theoretical advances are reviewed. A particular emphasis is a description of the development of reduced dimensional theories which can highlight chemical reactions that are likely to be of interest for more accurate quantum reactive scattering studies and new experiments. Furthermore, the reduced dimensional models allow for the development and testing of computationally inexpensive procedures that enable calculations to be performed on quantum effects in reactions of larger polyatomic molecules.
\end{abstract}




\section{Introduction}

The revolution in quantum mechanics pioneered by Schrodinger, Dirac, Heisenberg and others in the 1920s was picked up quite quickly by physical chemists in a Faraday Discussion on Molecular Spectra and Molecular Structure in $1929^{1}$. In that Discussion, papers by early practitioners of molecular quantum mechanics such as Lennard-Jones ${ }^{2}$, Mulliken $^{3}$ and Hund ${ }^{4}$ demonstrated that the valence-bond and molecular orbital theories were already explaining in detail the spectra of diatomic molecules and the exquisite link between molecular quantum mechanics and experimental observation was emerging. There was also a lively discussion on the appropriate quantum number notation for diatomic molecules and the term predissociation was introduced to the physical chemistry community by Henri ${ }^{5}$. In addition there was an historic paper by $\mathrm{C} \mathrm{V}$ Raman on the famous effect that bears his name ${ }^{6}$.

There have also been several Faraday Discussions largely devoted to reaction kinetics or dynamics that have involved the use of quantum mechanics to explain experimental results. The Discussion on Reaction Kinetics held in Manchester in 1937 addressed several challenges still of interest today ${ }^{7}$. The classic paper by Wigner ${ }^{8}$ on the transition state method discussed quantum tunnelling and concluded "It seems, therefore, that apart from reactions involving $\mathrm{H}$, the tunnelling effect cannot be made responsible at ordinary temperatures for any large decrease of the temperature independent factor." Many other notables of reaction kinetics and dynamics theory were present including Eyring and Michael Polanyi, and it should be noted also some of the legendary names of physical chemistry were there such as Brønsted, Delbruck, Hammett, Langmuir and Ingold. The first paper by Eyring ${ }^{9}$ summarised the status of electronic structure theory for calculating activation energies for chemical reactions - a field that has made huge strides since that time. There were also additional papers by Eyring ${ }^{10}$ and Evans ${ }^{11}$ on transition state theory, and a second paper by Evans and Polanyi ${ }^{12}$ on qualitative features of potential energy surface computation. There was a paper, highly relevant to 
this Faraday Discussion, by Bell ${ }^{13}$ which stated "So far no evidence has been produced which directly demonstrates the presence of the tunnel effect."

In 1991 there was a Faraday Discussion held in Nottingham on Structure and Dynamics of Reactive Transition States which gave a glimpse of the new experimental and theoretical studies that were being undertaken to directly study transition states ${ }^{14}$. Several of the methods and results I discuss below link to this forward-looking Faraday Discussion. Many subsequent Faraday Discussions also had papers connected to the theory of reaction kinetics or dynamics but it was the 1998 meeting, also held like the current one in Scotland, which was the first Discussion purely devoted to Chemical Reaction Theory ${ }^{15}$. This featured a Spiers Memorial lecture by Miller on "The Quantum and semiclassical theory of chemical reaction rates" ${ }^{16}$. Miller emphasised the progress made since the 1937 Faraday Discussion, in particular mentioning the improvement in $a b$ initio methods for calculating potential energy surfaces, development of Quantum Reactive Scattering (QRS) theory, and experimental methods based on molecular beams and lasers for studying detailed reaction dynamics. Then last year there was a further Faraday Discussion on Reaction Rate Theory in Cambridge which had a particular emphasis on recent developments in tunnelling theories for calculating rate constants ${ }^{17}$.

The current Faraday Discussion on Quantum Effects in Small Molecular Systems has papers which can be divided into three main areas: reaction dynamics, spectroscopy and molecules in confinement. It should be emphasised that "quantum effects" in this Faraday Discussion refers to effects involving the nuclei and not those involving purely the properties of electrons or electronic structure. This Spiers Memorial Lecture will concentrate on developments in the first of these topics - the quantum dynamics of chemical reactions. Three main features on quantum effects in chemical reactions that have been theoretically predicted and experimentally observed will be emphasised. These are quantum scattering resonances, quantum state-selective effects and 
tunnelling. It should be noted that a comprehensive review of developments in the spectroscopy of small molecules and clusters has been given very recently by Hochlaf ${ }^{18}$. 


\section{Quantum Scattering Resonances}

Quantum scattering resonances in chemical reactions have long been of interest to theoreticians but have only relatively recently been experimentally measured. They first showed up in QRS calculations on simple atom-diatom reactions of reduced dimensionality as structure in reaction probability plots against translational energy ${ }^{19,20}$. A good example is in Figure 1 where a QRS calculation for the collinear $\mathrm{F}+\mathrm{H}_{2} \rightarrow \mathrm{HF}+\mathrm{H}$ reaction by Schatz, Bowman and Kuppermann ${ }^{20}$ is shown.

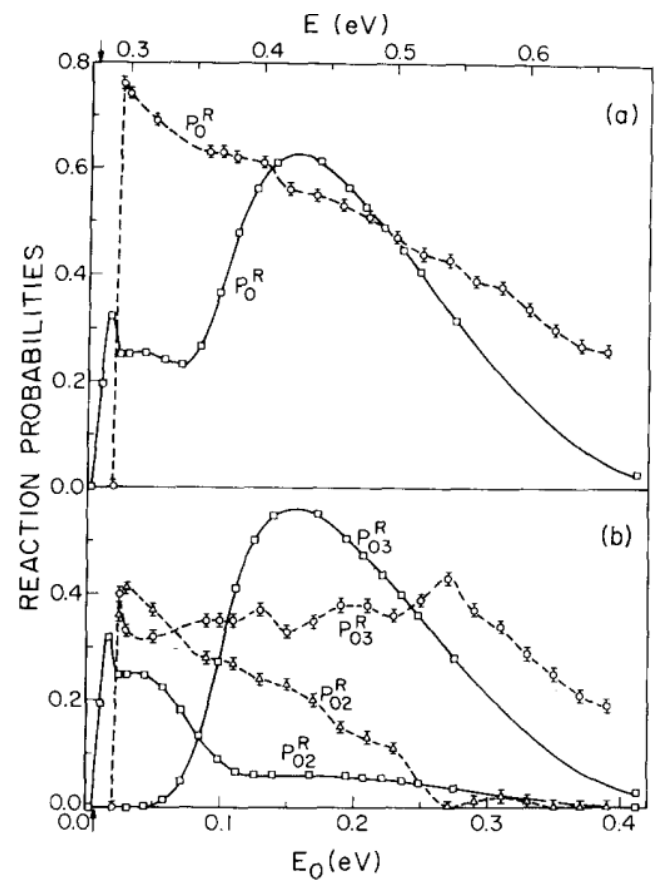

Fig 1. Quantum reaction probabilities for the collinear $\mathrm{F}+\mathrm{H}_{2}$ reaction as a function of the relative kinetic energy $E_{0}$. The top figure gives the total reaction probability while the bottom figure gives the probabilities for particular final vibrational states. Solid lines are quantum calculations and dotted lines quasiclassical trajectory results ${ }^{20}$.

Structure in the reaction probabilities can be seen which do not show up in quasiclassical trajectory (QCT) calculations which are also shown in the figure. A semiempirical potential energy surfaces due to Muckerman ${ }^{21}$ was used in these calculations. This paper and similar ones ${ }^{19,22}$ on the origin and predictions of such structures gave an emphasis to the study of the detailed dynamics of the $\mathrm{F}+\mathrm{H}_{2}$ reaction which has continued to the present day. 
A quantitative explanation of quantum reactive scattering resonances is nicely explained, calculated and portrayed by using hyperspherical coordinates ${ }^{23,24}$. Figure 2 shows hyperspherical coordinates for the same collinear $\mathrm{F}+\mathrm{H}_{2}$ reaction on the top of the potential energy surface ${ }^{25}$. The hyperradius is $\rho$ and the polar angle is $\alpha$.

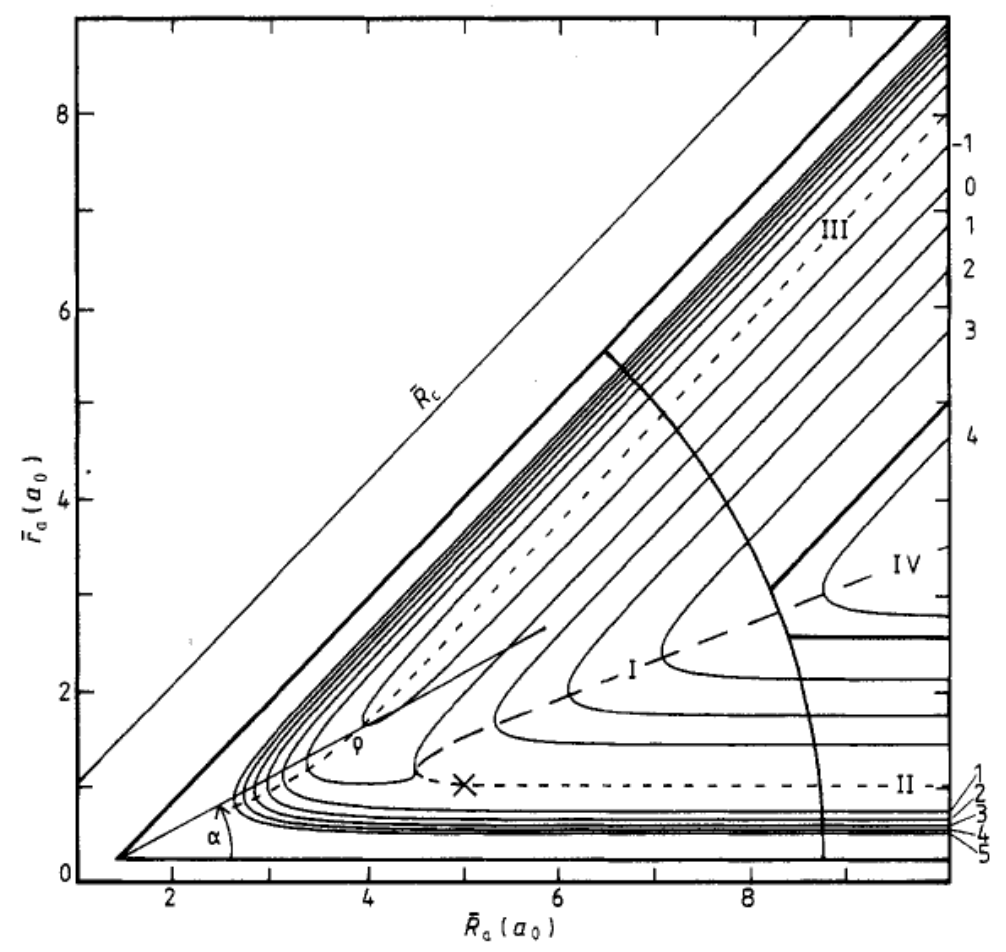

Fig 2. $\mathrm{FH}_{2}$ potential energy surface plotted in the hyperspherical radius $\rho$ and polar angle $\alpha^{25}$. The cross indicates the saddle-point position. Energy contour refer to the bottom of the $\mathrm{F}+\mathrm{H}_{2}$ valley at large distances and are in eV.

The quantum scattering calculations ${ }^{25}$ are done by expanding the wavefunction in basis functions in the angle $\alpha$ while numerically integrating along the hyperradius $\rho$. This gives a set of eigenstates $\varepsilon(\rho)$ on a grid of $\rho$ values which, for large values of $\rho$, tend to the vibrational states of the $\mathrm{H}_{2}$ reactant or the HF product. The plot of the eigenvalues of these eigenstates as a function of $\rho$ are called hyperspherical adiabats. Application of scattering boundary conditions gives the $\mathrm{S}$ matrix elements and reaction probabilities for reactive scattering between the reactant and product vibrational states. These reaction probabilities are shown in the bottom section of Figure 3 while the 
hyperspherical adiabats are shown at the top. The wells in the hyperspherical adiabats support bound states which correspond quantitatively to the resonance peaks in the reaction probabilities plotted against reaction energy. Plots of the eigenstates also show localized wavefunctions in the transition state region at the resonance energies.

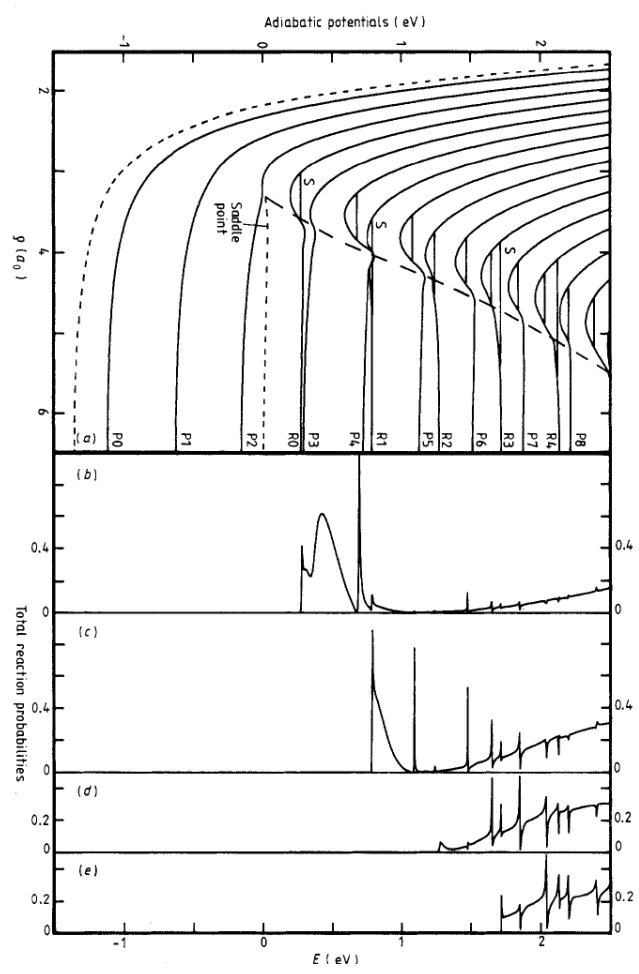

Fig 3. (a) Adiabatic energy potential energy curves $\varepsilon(\rho)$ for $\mathrm{FH}_{2}$. (b)- (d) Reaction probability plots for different initial vibrational states of $H_{2}(n)$ with $n=0-3^{25}$.

The resonance states have also been noticed in reduced-dimensionality calculations on several other simple atom-diatom reactions such as $\mathrm{H}^{+} \mathrm{H}_{2}{ }^{26}, \mathrm{O}+\mathrm{H}_{2}{ }^{27}$ and $\mathrm{F}+\mathrm{HBr}^{28}$. The clear theoretical prediction of reactive scattering resonances presented a challenge for their observation to reaction dynamics experiments. As the resonance peaks are a very narrow function of collision energy, when the reaction probabilities are averaged over collision energy they do not show a clear influence on the most commonly measured observable for chemical reactions - the reaction rate constant. Thus it was necessary to look to more detailed reaction dynamics experiments to observe them. 
A key aspect here was the development of QRS methods for atom-diatom reactions in three dimensions ${ }^{30}$ together with the construction of accurate potential energy surfaces from the best $a b$ initio computations ${ }^{31}$. The predictions from such calculations should give highly reliable results for comparisons with experiment, all the way from the quantum state-selective differential cross sections as a function of collision energy to rate constants as a function of temperature. From the late 1970 s onwards there was a continual progress in such QRS techniques involving the solution of both the timeindependent and time-dependent Schrödinger equation for the quantum scattering process $^{29,32-36}$. This included the use of the hyperspherical coordinates in three dimensions ${ }^{37}$ and the construction of a general computer code using them for $A+B C$ reactions in $3 D^{38}$.

The use of wavepackets to solve the time-dependent Schrödinger equation also had major improvements culminating in the converged calculations of differential cross sections (DCS) for reactions such as $\mathrm{F}+\mathrm{H}_{2}$ on accurate potential surfaces ${ }^{39,40}$. This progress also enabled detailed effects of isotopic substitution of $\mathrm{H}_{2}$ to be studied. Figure 4 shows a typical calculation on the $3 \mathrm{D} F+\mathrm{HD}$ reaction ${ }^{41}$. These reaction cross sections plotted as a function of collision energy show a clear peak due to a quantum resonance which is not obtained in a QCT computation.

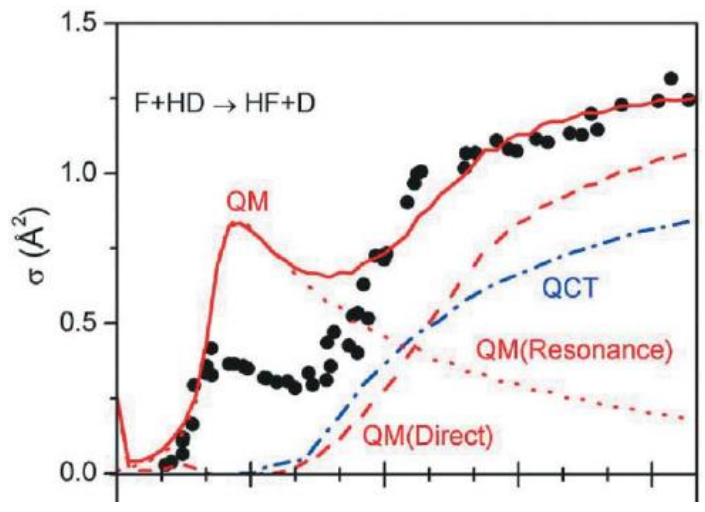

Fig 4. Integral cross sections as a function of collision energy, of the two isotopic product channels in the $F$ + HD reaction. The experimental results (solid dots) are normalized to QCT and QRS results by a single scaling factor for both channels ${ }^{41}$. 
This effort was matched by experimental progress, in particular using molecular beams combined with $\mathrm{H}$-atom Rydberg tagging time-of-flight or time-sliced velocity mapped imaging techniques ${ }^{42}$. The 3D QRS calculations on the $\mathrm{F}+\mathrm{H}_{2}$ reaction suggested that the resonances would show up most clearly in differential cross section measurements for particular scattering angles and collision energies. Figure 5 shows such a comparison between theory and experiment for the $\mathrm{F}+\mathrm{H}_{2}$ reaction ${ }^{43}$ and the agreement is excellent.
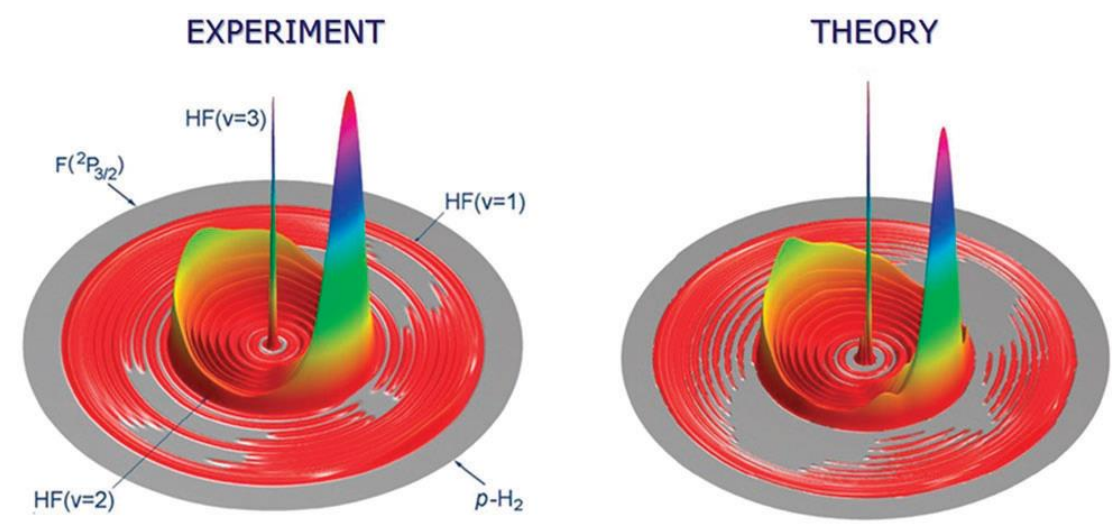

Fig 5. Experimental and QRS three-dimensional contour plots for the HF product translational energy and angular distributions of the $\mathrm{F}+\mathrm{H}_{2}(\mathrm{j}=0)$ reaction at a collision energy of $0.52 \mathrm{kcal} \mathrm{mol}^{-1}$. Different rings represent different $\mathrm{HF}$ product rovibrational states ${ }^{43}$.

The sharp peaks in the plots provide clear theoretical and experimental evidence for the reactive scattering resonances. Subsequent studies on the F+HD reaction also show novel structures associated with the resonances associated with initial vibrational excitation in the HD reagent ${ }^{44}$. Figure 6 shows the backward scattering in the DCS for $\mathrm{HD}$ in the $\mathrm{v}=0$ and 1 states together with plots of the reactive scattering wavefunctions which give rise to the resonances ${ }^{45}$. 

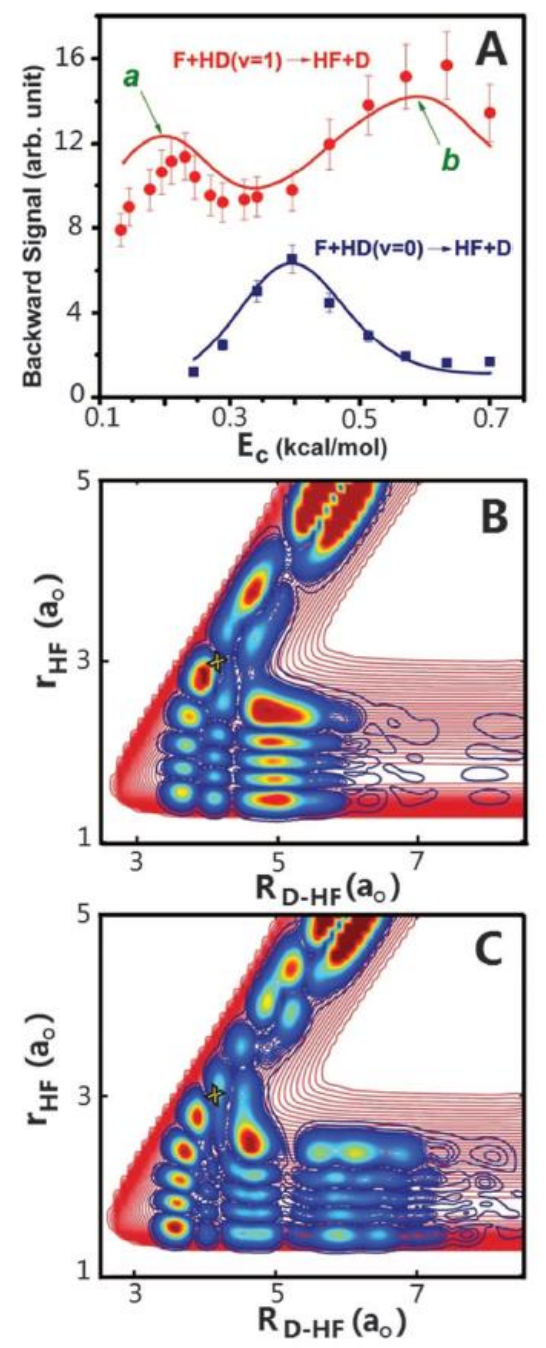

Fig. 6. A. Experimental (solid circles and squares) and QRS (solid lines) DCS for the backward scattered HF products of the $F+H D(v=1, j=0)$ reaction (red line and circles) and the $F+H D$ reaction $(v=0, j=0)$ (blue line and squares), over a range of collision energies $E_{c}$. $B$ and $C$ give plots of the wavefunctions at the energies $a$ and $b$ of the two quantum scattering resonances shown in plot $A^{45}$.

What is clear from these results is that the resonance positions and their influence on the DCS are very sensitive to the detailed topology of the potential energy surface and only when the surface is accurate, and the QRS calculations are converged in all dimensions, will there be a precise quantitative agreement between theory and experiment.

Another type of experiment which can detect reactive scattering resonances involves the spectroscopy of the photodetachment of anions ${ }^{46}$. Yet again, $\mathrm{F}+\mathrm{H}_{2}$ has been a good 
example for both theory and experiment. In this case, starting from the stable anion $\mathrm{FH}_{2}{ }^{-}$ and detecting the energy of the electrons produced after photodetachment enables the dynamics of the $\mathrm{FH}_{2}$ transition state to be accessed. Theory again provided predictions which were subsequently measured in experiment. In a paper entitled "How to observe the elusive resonances in $\mathrm{F}+\mathrm{H}_{2}$ reactive scattering" Russell and Manolopoulos ${ }^{47}$ reported a calculation of the spectrum for the photodetachment of $\mathrm{FH}_{2}^{-}$by overlapping the wavefunction for $\mathrm{FH}_{2}{ }^{-}$with that for the $\mathrm{F}+\mathrm{H}_{2} \rightarrow \mathrm{HF}+\mathrm{H}$ reactive scattering calculated using the time-dependent wavepacket technique. Structure was calculated in the photodetachment spectrum at the energies for which resonances were also obtained. The fine width of the resonances proved a real challenge to their observation in photodetachment experiments at that time but success has recently been achieved ${ }^{48}$. The use of slow-electron velocity-map imaging and cryogenic cooling of the ions gives photoelectron spectra with sub-meV resolution. This has allowed the observation of the structure predicted in the spectra for both $\mathrm{FH}_{2}{ }^{-}$and $\mathrm{FD}_{2}{ }^{-}$. An example is given in Figure 7 for $\mathrm{FH}_{2}^{-}$.

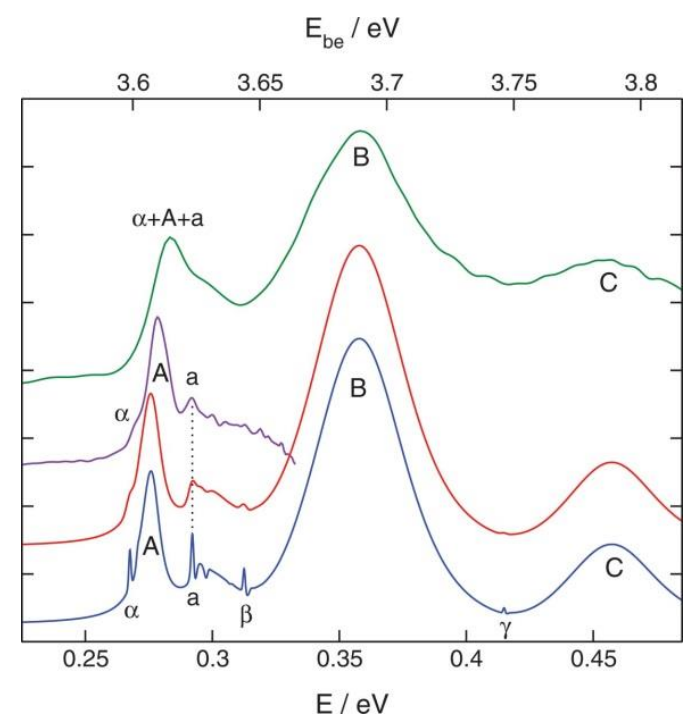

Fig 7. Photodetachment spectra of $\mathrm{FH}_{2}{ }^{-}$. The experimental overview spectra (10 meV resolution) are shown in green, while the highest resolution ( 2 to $3 \mathrm{meV}$ ) experimental taken over narrower energy windows are shown in purple. QRS results are shown at $1 \mathrm{meV}$ energy resolution (blue) and $3 \mathrm{meV}$ resolution $(\text { red })^{48}$. 
The success of the theory and experiment for photodetachment of triatomic molecular ions suggests that it will be of interest to perform similar studies on more complicated polyatomic species. A good example here is $\mathrm{FCHD}_{3}{ }^{-}$. Calculations using a reduced dimensionality method ${ }^{49}$ on the $\mathrm{F}+\mathrm{CHD}_{3}$ reaction showed many resonances in the reaction probability plots as shown in Figure 8.
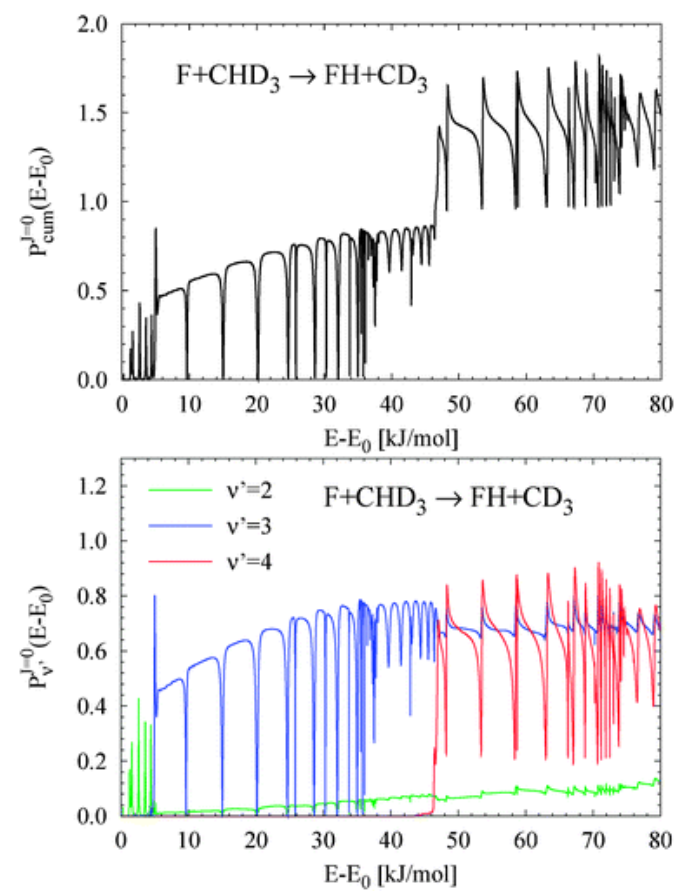

Fig. 8. Cumulative (upper panel) and product $\mathrm{HF}$ vibrational selected reaction probabilities of the $\mathrm{F}+\mathrm{CHD}_{3}$ reaction ${ }^{49}$ (lower panel). The calculations were performed with reduced-dimensionality QRS.

Recently photodetachment spectra for $\mathrm{FCH}_{4}{ }^{-}$and $\mathrm{FCD}_{4}{ }^{-}$were observed using the highresolution methods and structure was again observed which can be identified with the calculated reactive scattering resonances of Manthe and co-workers as shown in Figure $9^{50}$. 

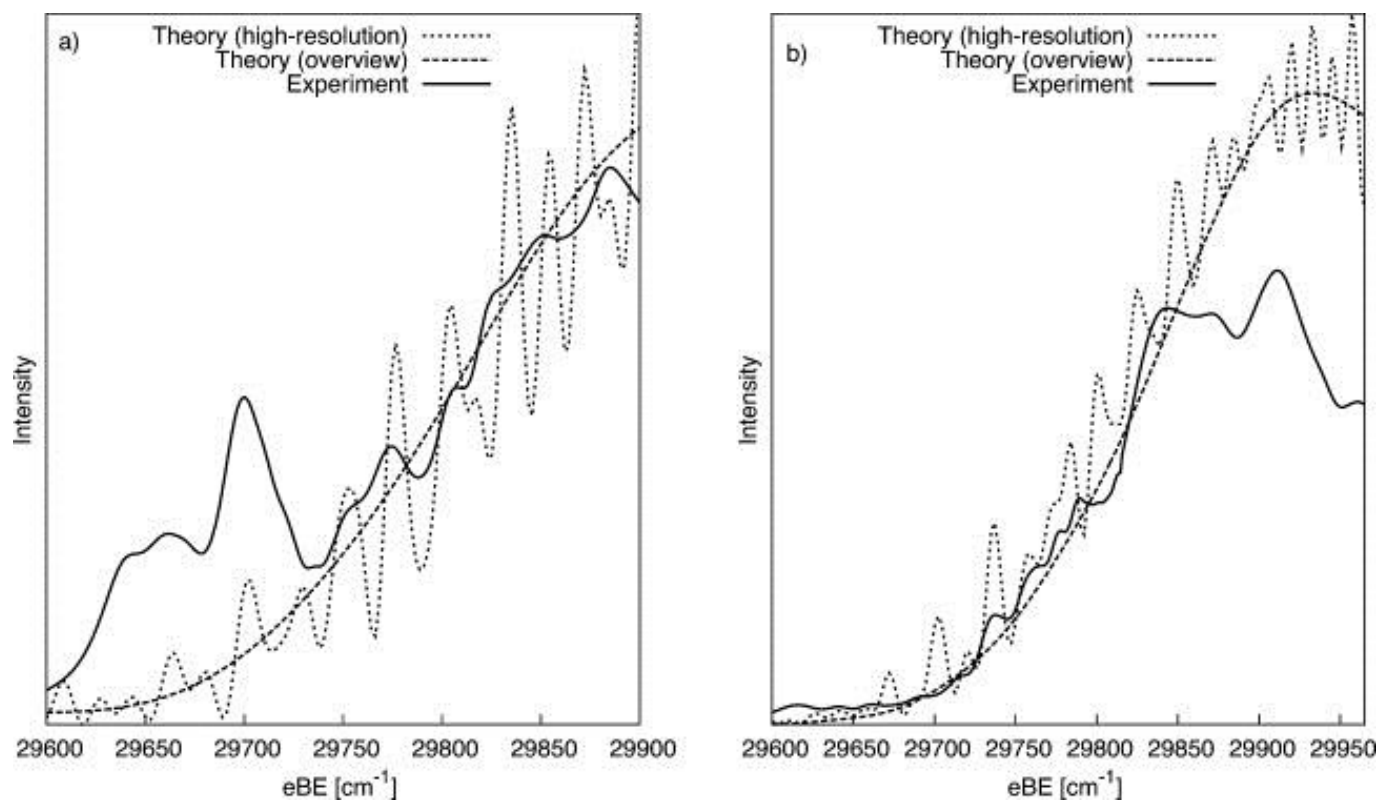

Fig. 9. Theoretical and experimental high-resolution photodetachment spectra ${ }^{50}$ for a) $\mathrm{FCH}_{4}{ }^{-}$and b) $\mathrm{FCD}_{4}{ }^{-}$.

The polyatomic nature of this system gives progressions of many more resonances over the three-atomic case and these will be very precise tests on the calculated potential energy surfaces. There has also been a very recent joint theoretical and experimental study on the challenging $\mathrm{CH}_{3} \mathrm{OHF}^{-}$ion where structure in the photodetachment spectrum was again identified with quantum reactive resonances ${ }^{51}$. The field of photodetachment transition state spectroscopy is clearly moving at a fast pace to larger polyatomic systems.

A system where quantum resonances has a direct chemical application is in the formation of $\mathrm{O}_{3}$ from the collision between $\mathrm{O}$ and $\mathrm{O}_{2}$ assisted by three body stabilization - a very important process in the earth's atmosphere. Reduced dimensional infiniteorder sudden calculations $s^{52,53}$ highlighted the importance of quantum scattering resonances for this process and even helped to explain isotopic fractionation effects. In this current Faraday Discussion Teplukhin and Babikov report results of three dimensional QRS calculations of the resonances for $\mathrm{O}+\mathrm{O}_{2}$ collisions obtained using the time-independent hyperspherical coordinate method $^{54}$. 


\section{Quantum State Selection Effects}

\section{3a. Vibrational effects in polyatomic reactions}

The effects of initial quantum state vibrational excitation on chemical reactions, and vibrational distributions of the product molecules, have been studied for many years in both theory and experiment. John Polanyi's Nobel Prize Lecture ${ }^{55}$ emphasised these features and related these effects to early or late barriers in potential energy surfaces for reactions of atoms with diatomic molecules. The Faraday Discussion on Kinetics of State-Selected Species held in Birmingham in 1977 also highlighted this field ${ }^{56}$. A more recent interest is to examine whether such vibrational state-selective effects appear in the reactions involving polyatomic molecules where different types of vibrations are involved. Yet again calculations with reduced dimensionality have proved useful and insightful ${ }^{57}$.

The first quantum dynamics calculations on four-atom reactions in reduced dimensionality showed vibrational state-selective effects which in some cases gave quantitative agreement with experiment ${ }^{57-60}$. Some of the first such results on the

$$
\mathrm{D}_{2}(\mathrm{v})+\mathrm{OH}(\mathrm{j}) \rightarrow \mathrm{DOH}(\mathrm{n}, \mathrm{m})+\mathrm{D}
$$

reaction were reported as part of the Faraday Discussion on Structure and Dynamics of Reactive Transition States ${ }^{61}$. The Rotating Bond Approximation (RBA) ${ }^{59}$ explicitly accounts for initial $\mathrm{OH}$ rotation $\mathrm{j}$ and $\mathrm{D}_{2}$ vibration $\mathrm{v}$, together with $\mathrm{DOH}$ bending and $\mathrm{D}$ $\mathrm{OH}$ stretching quantum numbers $(n, m)$ respectively. The theory predicted ${ }^{62}$ the product with $\mathrm{m}=2$ quanta in the $\mathrm{D}-\mathrm{OH}$ stretch would be preferred with somewhat smaller cross sections for product $\mathrm{DOH}(0,1)$ and $(1,1)$. This prediction was subsequently confirmed in experiments done by Davis and co-workers ${ }^{63,64}$ (see Figure 9). 


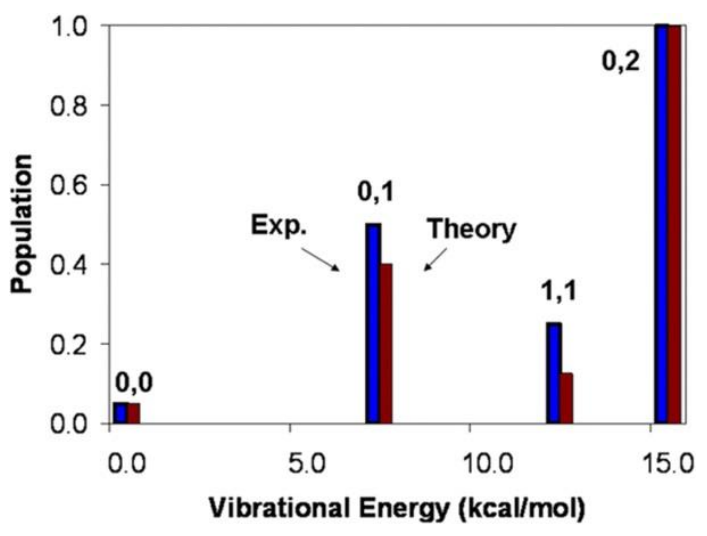

Fig. 9. Predicted ${ }^{62}$ and measured ${ }^{63}$ vibrational product distributions for the $D_{2}(v)+O H(j) \rightarrow D O H(n, m)+D$ reaction at a collision energy of $6.3 \mathrm{kcal} / \mathrm{mol}$. The integer labels $n, m$ are the DOH bending $(n)$ and OD stretching $(m)$ vibrations of product $\mathrm{DOH}^{64}$.

More recently QRS wavepacket computations in all dimensions have been carried out on the $\mathrm{OH}+\mathrm{H}_{2}$ reaction and isotopes of $\mathrm{H}$ with accurate potential energy surfaces obtained from high-quality $a b$ initio calculations ${ }^{65-67}$. These computations also give similar good comparison to experiment for vibrational product distributions and also have provided the first accurate differential cross sections for this reaction ${ }^{66,67}$. An example for the $\mathrm{HD}+\mathrm{OH}$ reaction is plotted in Figure 10 and very good agreement between theory and experiment is obtained ${ }^{66}$.
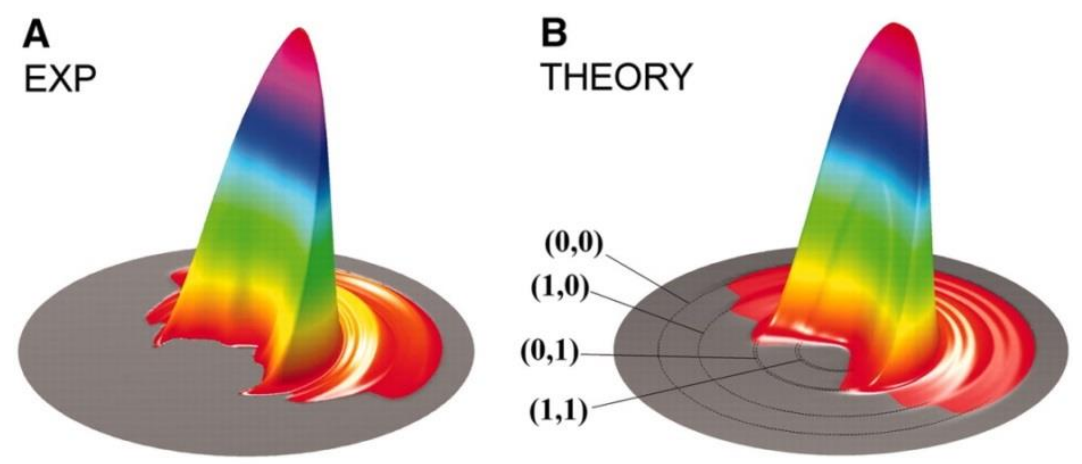

Fig. 10. Experimental (A) and theoretical (B) surface plots for the product translational energy and angle distributions for the $\mathrm{HD}+\mathrm{OH}$ reaction at the collision energy of $6.9 \mathrm{kcal} / \mathrm{mol}$. Dashed circles in (B) represent the maximum translational energies of the products in the indicated $\mathrm{H}_{2} \mathrm{O}$ product vibrational states, which are labelled by $(n, m)$, where $n$ is the quantum number for the bending mode and $m$ for the $\mathrm{OH}$-stretching modes ${ }^{66}$. 
QRS wavepacket calculations on state-selective effects in several other polyatomic reactions have been reported including the reactions of $\mathrm{H}_{2} \mathrm{O}$ with $\mathrm{F}^{68}, \mathrm{Cl}^{69}$ and $\mathrm{O}\left({ }^{3} \mathrm{P}\right)^{70}$, and $\mathrm{H}$ reacting with $\mathrm{H}_{2} \mathrm{~S}^{71}$ and $\mathrm{CO}_{2}{ }^{72}$. Going beyond four atoms, $\mathrm{H}+\mathrm{CH}_{4}$ has also become a major example with several different types of approximate and accurate methods being applied ${ }^{74-77}$. The Multi-Configurational Time Dependent Hartree (MCTDH) calculations of Manthe and co-workers have given benchmarks for testing the accuracy of more approximate methods for this reaction ${ }^{78,79}$. Symmetry effects and product distributions for the $\mathrm{H}+\mathrm{CHD}_{3}$ reaction is the subject of the paper by Ellerbrock and Manthe and co-workers presented at this Faraday Discussion ${ }^{80}$. A recent review summarises the impressive recent progress in QRS calculations on polyatomic reactions over the last ten years ${ }^{36}$.

\section{3b. Rotational Effects on Chemical Reactions}

Understanding the effects of initial rotational quantum states on chemical reactions is of considerable interest as this links to reaction rate constants and their temperature dependence. A different type of reaction becomes important here - fast reactions without barriers in the potential energy surfaces as shown in figure 11.

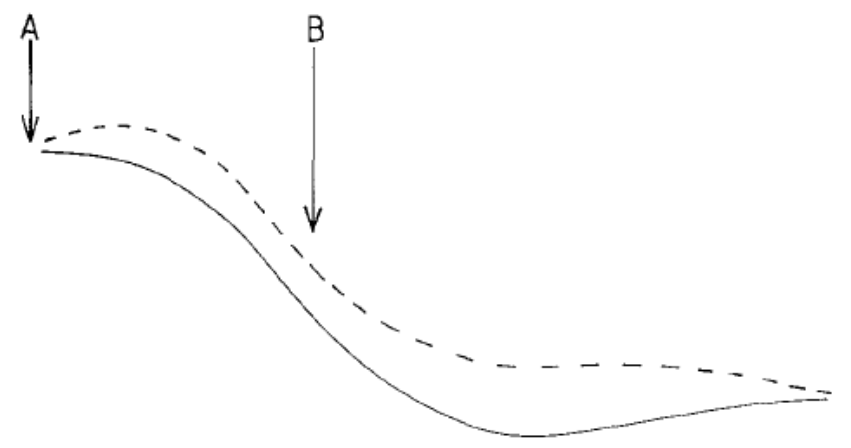

Fig 11. Potential curve for a fast reaction with no barrier in the potential surface (unbroken line). The dotted line shows the effective potential with a centrifugal term included ${ }^{81,83}$. 
Here again simple theories are useful. Quantum tunnelling is not important here, except perhaps in the limit close to temperature $T=0$, but quantum rotational effects can be significant.

A QRS-based Rotationally Adiabatic Capture (RAC) theory ${ }^{81-88}$ was developed to treat this type of fast reaction. This theory involves the following procedures ${ }^{83}$ :

1) Construct the longer-range part of the potential energy surface in the entrance channel to the reaction using the multipolar expansion and monomer properties. 2) Diagonalise this potential on a grid of intermolecular distances $R$ using an appropriate rotational basis set. This produces a set of rotationally adiabatic potential curves corresponding with well-defined rotational states for large R.

3) Add the centrifugal potential labelled by the total angular momentum J to the rotationally adiabatic curves. Assign a reaction probability of unity for all J values with maxima in the effective potentials below the collision energy. This gives very simple formulae for the reaction cross sections ${ }^{82,83}$.

4) Boltzmann average the reaction cross sections over collision energies to produce rate constants labelled in the initial rotational states $\mathrm{k}(\mathrm{j})$.

Particularly strong effects of initial rotational states $j$ on the rate of chemical reactions $k(j)$ were predicted for ion-molecule reaction with this RAC theory such as is shown in Figure 12 for $\mathrm{N}^{+}$with $\mathrm{NH}_{3}{ }^{87}$. 


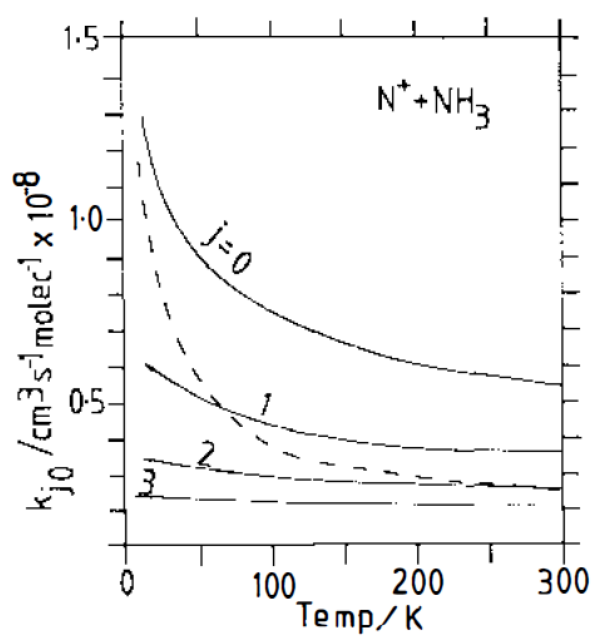

Fig. 12. Rotationally selected rate constants $\mathrm{k}(\mathrm{j})$ for the $\mathrm{N}^{+}+\mathrm{NH}_{3}(\mathrm{j}, \mathrm{k}=0)$ reaction calculated by using the rotationally adiabatic capture theory ${ }^{83}$. The dotted line shows the rate constant $k(T)$ obtained by Boltzmann averaging over the $\mathrm{k}(\mathrm{j})^{87}$.

Increasing $\mathrm{T}$ brings in more and more j states with lower rate constants. Accordingly, the $k(j)$ effect produces quite a strong negative temperature dependence on the overall reaction rate constant $k(T)$. Figure 13 gives a good example of this negative temperature effect predicted ${ }^{87}$ for the reactions of $\mathrm{H}_{2} \mathrm{O}$ with ions which gives good comparison with experimental rate constants down to $27 \mathrm{~K}$.

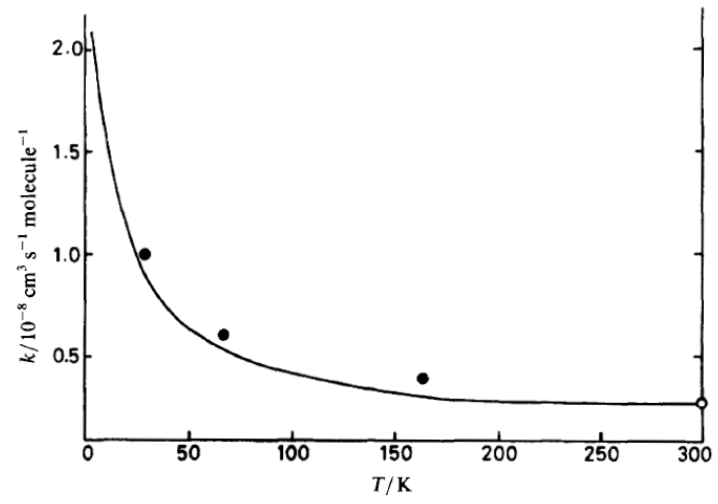

Fig 13. Calculated rate constants (solid line) for $\mathrm{N}^{+}+\mathrm{H}_{2} \mathrm{O}$ compared ${ }^{87}$ with experimental results reported in J. B. Marquette, B. R. Rowe, G. Dupeyrat, G. Poissant and C. Rebrion, Chem. Phys. Lett., 1985, 122, 431435(solid circles) and D. Smith, N. G. Adams and T. M. Miller, J. Chem. Phys., 1978, 69, 308-318 (open circle). 
These predictions have relevance for understanding and simulating the chemistry of cold interstellar clouds where the temperatures can be as low as $20 \mathrm{~K}$ and only fast barrier-less chemical reactions can occur ${ }^{89}$.

Despite the fact that many experimental measurements of rate constants $k(T)$ for fast reactions of both neutral and ionic species compare very well with rotational adiabatic theory calculations ${ }^{81-88,90,91}$ and other theories ${ }^{92}$ it is only very recently that a conclusive experiment has been carried out to demonstrate the predicted $k(j)$ effect. Kilaj et $\mathrm{al}^{93}$ devised an experiment with electrostatic deflection of a molecular beam that separated ortho and para $\mathrm{H}_{2} \mathrm{O}$ molecules which were reacted with cold $\mathrm{N}_{2} \mathrm{H}^{+}$molecules in an ion trap, as shown in Figure 14.

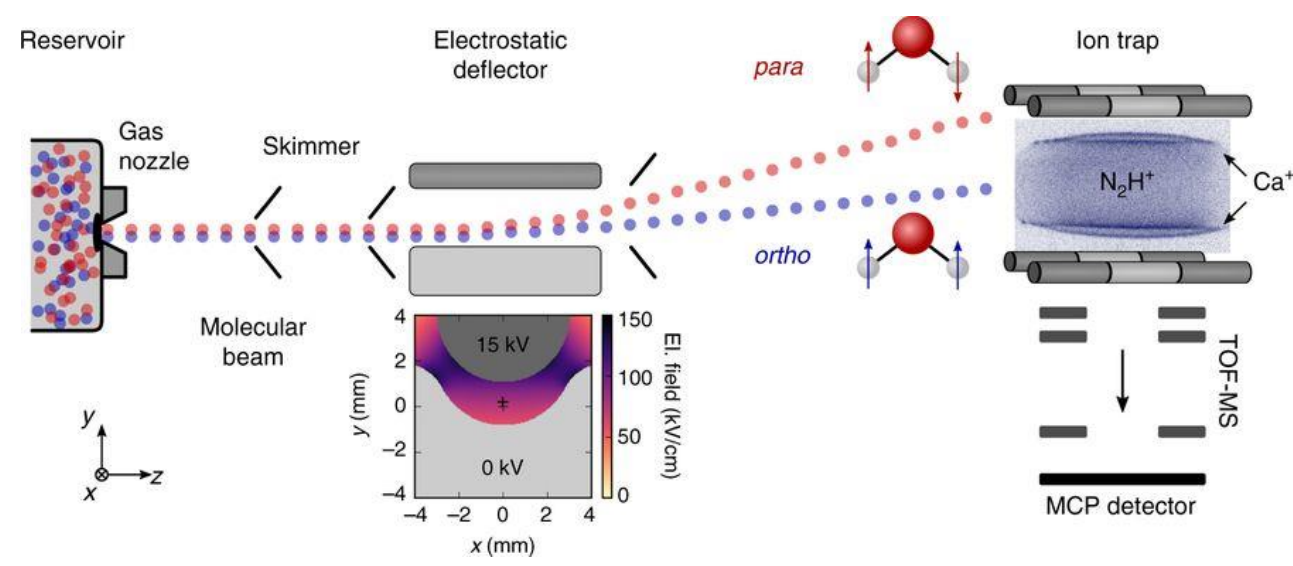

Fig. 14. Schematic of the experimental setup of Kilaj et $\mathrm{al}^{93}$ separating ortho and para $\mathrm{H}_{2} \mathrm{O}$ and reacting with $\mathrm{N}_{2} \mathrm{H}^{+}$. https://creativecommons.org/licenses/by/4.0/

As ortho and para $\mathrm{H}_{2} \mathrm{O}$ have even or odd rotational states and the temperature of the beam was very low, the reactant $\mathrm{H}_{2} \mathrm{O}$ molecules were in $\mathrm{j}=0$ or 1 states. It was found ${ }^{93}$ that the $\mathrm{j}=0$ rate constants were $20 \%$ larger than those for $\mathrm{j}=1$ and the absolute rates were in quantitative agreement with those calculated by the rotationally adiabatic capture theory. 
The paper by Gianturco and co-workers in this Faraday Discussion on quantum dynamics and experimental collisional relaxation of ortho and para $\mathrm{NH}_{2}{ }^{-}$under photodetachment in cold ion traps links to the above study ${ }^{94}$. 


\section{Rate Constants}

Rate constants have a special role as they are the fundamental kinetic quantities of molecular science and are needed to understand many chemical processes. Therefore, the development of general, robust and accurate rate constant theories is an area of considerable importance. Ever since the 1937 Faraday Discussion on Reaction Kinetics, transition state theory (TST) has been central. With the progress of $a b$ initio quantum chemistry which can directly provide the quantities needed, namely barrier heights and vibrational frequencies of the transition state and reactants, modern forms of TST have become hugely powerful ${ }^{95}$. However, conventional TST has a major drawback that it neglects quantum tunnelling and, especially for reactions involving $\mathrm{H}$ atoms, this can have large effects on reactions. Therefore, developing efficient methods to treat quantum tunnelling is a major topic for the theoretical reaction dynamics community.

QRS calculations not only give reaction dynamics quantities but can also provide rate constants over a wide temperature range if the calculations are performed over the necessary number of partial waves J, initial quantum states and translational energies. They therefore provide valuable benchmarks ${ }^{29}$. Much of the early work was on the classic $\mathrm{H}+\mathrm{H}_{2}$ reaction ${ }^{30}$ where quantitative agreement between theory and experiment was eventually obtained ${ }^{32}$ and in more recent years the $\mathrm{OH}+\mathrm{H}_{2}$ and $\mathrm{H}+\mathrm{CH}_{4}$ reactions have become benchmark systems ${ }^{57-60,73-81}$.

Figure 15 shows a comparison of many calculations of rate constants performed for the $\mathrm{H}+\mathrm{CH}_{4}$ reaction ${ }^{96}$. It can be seen that the classical TST rates with no tunnelling give rate constants an order of magnitude less than the rates obtained with theories that include tunnelling. 


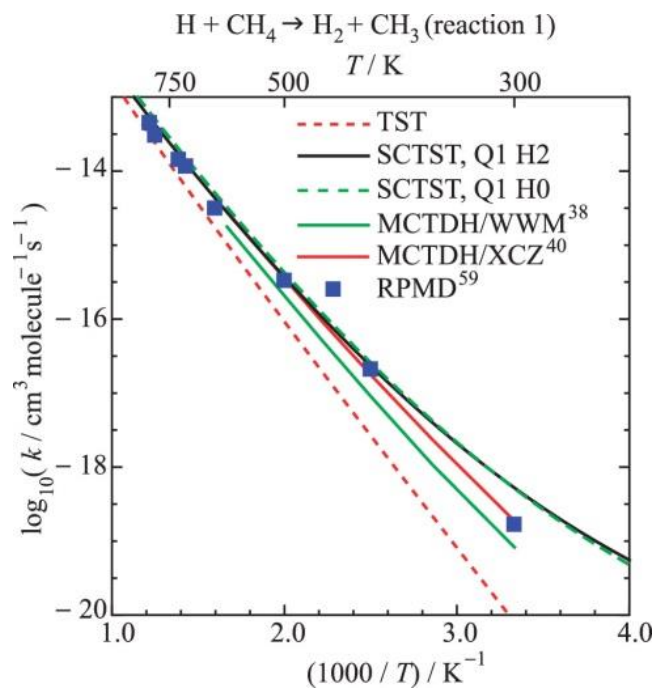

Fig 15. Rate constants for $\mathrm{H}+\mathrm{CH}_{4}$ calculated by Semiclassical Transition State Theory (SCTST) ${ }^{96}$ using two different sets of anharmonic constants presented in comparison with results from $\mathrm{MCTDH}^{79}$ and Ring Polymer Molecular Dynamics (RPMD) ${ }^{101}$ calculations. Results from conventional TST are the dashed red curve.

It should also be mentioned that as the rate constants depend exponentially on the height of the reaction barrier, highly accurate $a b$ initio calculations need to be employed. Furthermore, it is only recently that sophisticated methods for applying the full symmetry of molecules taking part in reactions have allowed for smooth and reliable potential energy surfaces to be obtained that accurately describe all reaction channels ${ }^{97}$. However, this approach still requires many computationally expensive $a b$ initio calculations, the required number of which goes up by orders of magnitudes as the dimensions of the reaction system increases. Similarly, the computational expense of QRS calculations increases in the same way. For this reason, the development of approximate but reliable theories which can compute rate constants for general polyatomic reactions with minimal computational expense is a very active area.

Going beyond TST, the variational TST approach combined with tunnelling path selections developed by Truhlar and co-workers ${ }^{98}$ has proved to be generally applicable and can be applied to systems as complex as reactions catalysed by enzymes ${ }^{99}$. In this approach, some judgement has to be made in the choice of the appropriate reaction 
path. Ring polymer molecular dynamics (RPMD) ${ }^{100-102}$ and related theories are proving to be useful approaches as they employ the method of path integrals to give a straightforward extension of well-established molecular dynamics computational procedures. Related to this work is the instanton method ${ }^{103-105}$ which provides approximate tunneling rate constants from the minimum-action path linking reactants to products at a given temperature. The paper by Richardson and co-workers at this Faraday Discussion highlights the instanton approach and discusses how to link it with high level $a b$ initio calculations ${ }^{106}$.

A simple and realistic method to account for quantum tunnelling in chemical reactions is the Semiclassical Transition State Theory (SCTST) developed by Miller and co-workers ${ }^{107-}$ ${ }^{8}$. This method requires just a force-field expansion up to fourth order of the potential energy about the transition state and does not require a full potential energy surface. The necessary parameters can be obtained almost directly from modern $a b$ initio quantum chemistry $\operatorname{codes}^{109}$. Reaction probabilities are computed using the WKB approximation. The first SCTST calculations of rate constants were done several years ago ${ }^{108}$ when there were not good QRS computations for comparison and the method was not widely taken up. However, recent developments in ab initio quantum chemistry derivative codes and comparison with QRS calculations have demonstrated that SCTST is much more accurate than was realised previously ${ }^{96,110-118}$.

A powerful and general QRS approximate method has been developed which can be applied to many polyatomic reactions ${ }^{74,76,77,119-127}$. This combined reduced-dimensional and quantum chemistry (RDQC) approach has been informed by the successful applications of reduced dimensionality methods which have already been highlighted in this paper. The RDQC computational procedure is as follows:

1) The chemical bonds being broken $\left(r_{1}\right)$ and formed $\left(r_{2}\right)$ in the reaction are converted into polar (hyperspherical) coordinates $(\rho, \delta)$ for which a set of grid points are chosen. 
2) For each fixed grid point the geometries of all other spectator degrees of freedom $\{\mathrm{s}\}$ are optimised using the MP2 electronic structure method and their vibrational frequencies $\left\{v_{\mathrm{s}}\right\}$ are computed.

3) For each grid point, the accurate $\operatorname{CCSD}(T)$ energy at the optimised geometry is computed.

4) The zero-point energies $\left\{v_{\mathrm{s}} / 2\right\}$ of the spectator modes are added to the $\operatorname{CCSD}(T)$ energies to produce a two-dimensional effective potential energy surface $V(\rho, \delta)$.

5) Time-independent QRS calculations in the $(\rho, \delta)$ hyperspherical coordinates are performed to calculate reaction probabilities for $\mathrm{V}(\rho, \delta)$ as a function of collision energy E.

6) The required rate constants $k(T)$ are computed from these probabilities by Boltzmann-averaging over $E$ and using the computed $\left\{v_{s}\right\}$ for the transition state and reactants.

This approach treats accurately the vibrationally adiabatic transition state barrier energy and only requires a minimum (typically about 30 ) of $\operatorname{CCSD}(T)$ ab initio points. As the QRS calculations are only two-dimensional the computer time needed for the quantum scattering part of the calculation is minimal. Furthermore there is no ad hoc reaction path needed in the RDQC method.

The RDQC method has been applied successfully to many reactions of polyatomics larger than has been possible with previous QRS computations. This includes the reactions of $\mathrm{H}$ atoms with $\mathrm{C}_{2} \mathrm{H}_{6}{ }^{120}, \mathrm{HCF}_{3}{ }^{121}$, cyclic ${ }^{122}$ and linear ${ }^{77} \mathrm{C}_{3} \mathrm{H}_{6}$ and $\mathrm{n}-\mathrm{C}_{4} \mathrm{H}_{10}{ }^{123}$; the heavy-light- heavy $\mathrm{H}$ atom transfer reaction between $\mathrm{CH}_{4}$ and $\mathrm{CH}_{3}{ }^{124}$, and the $\mathrm{H}$ atom transfer reaction in $\mathrm{Cl}+\mathrm{CH}_{4}{ }^{125}$ including the treatment of the spin-orbit states of $\mathrm{Cl}^{126,127}$. In all cases when reliable experimental rate constant data are available the comparisons have been good. In addition, the method has also provided reaction dynamics quantities such as vibrational state-selected integral and differential cross sections which have allowed for extra comparisons with experiment ${ }^{125-127}$. 
Figure 16 shows typical rate constants calculated with the RDQC method for the reaction of $\mathrm{H}$ atoms with cyclopropane ${ }^{122}$. Also shown is the two-dimensional effective potential energy surfaces $\mathrm{V}(\rho, \delta)$ for this reaction. It can be seen that the agreement with experiment for this important reaction of a hydrocarbon is very good. It can also be noticed that the classical TST rate constants become well over an order of magnitude lower than the RDQC values for temperatures below 300K emphasising again the large quantum tunnelling effects in this type of reaction.
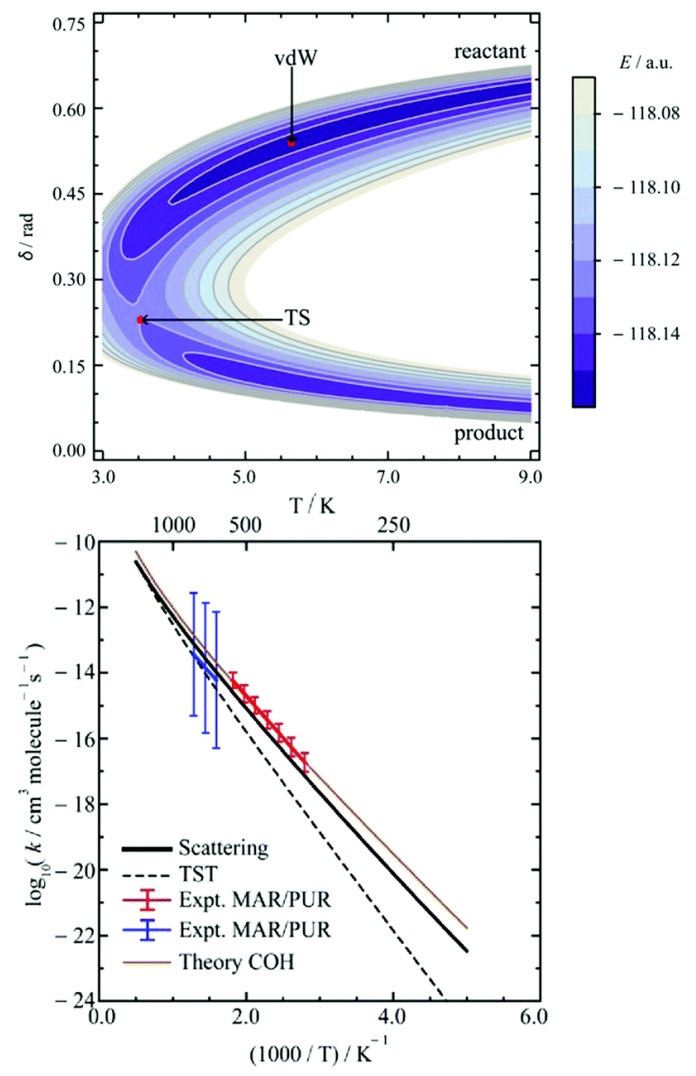

Fig. 16. (Top) 2D PES for the $\mathrm{H}+$ cyc- $\mathrm{C}_{3} \mathrm{H}_{6} \rightarrow \mathrm{H}_{2}+\mathrm{cyc}-\mathrm{C}_{3} \mathrm{H}_{5}$ reaction. (Bottom) Comparison of scattering (RDQC), TST and experimental reaction rate constants for the $\mathrm{H}+\mathrm{cyc}-\mathrm{C}_{3} \mathrm{H}_{6} \rightarrow \mathrm{H}_{2}+\mathrm{cyc}-\mathrm{C}_{3} \mathrm{H} 5$ reaction (see Ref 122 for more details).

The RDQC approach has been useful in the testing of the accuracy of the SCTST. In every case considered to date ${ }^{116-118,130}$, including reactions such as $\mathrm{H}$ atoms with $\mathrm{CH}_{4}, \mathrm{C}_{2} \mathrm{H}_{6}$, $\mathrm{C}_{3} \mathrm{H}_{6}$, and even the heavy-light- heavy $\mathrm{H}$ atom transfer reaction between $\mathrm{CH}_{4}$ and $\mathrm{CH}_{3}$, the comparison of the SCTST rate constants with the QRS values has been shown to be 
excellent. Examples of this agreement are given in Figure 17 for the $\mathrm{H}$ exchange reaction in $\mathrm{CH}_{4}+\mathrm{CH}_{3}{ }^{116}$.
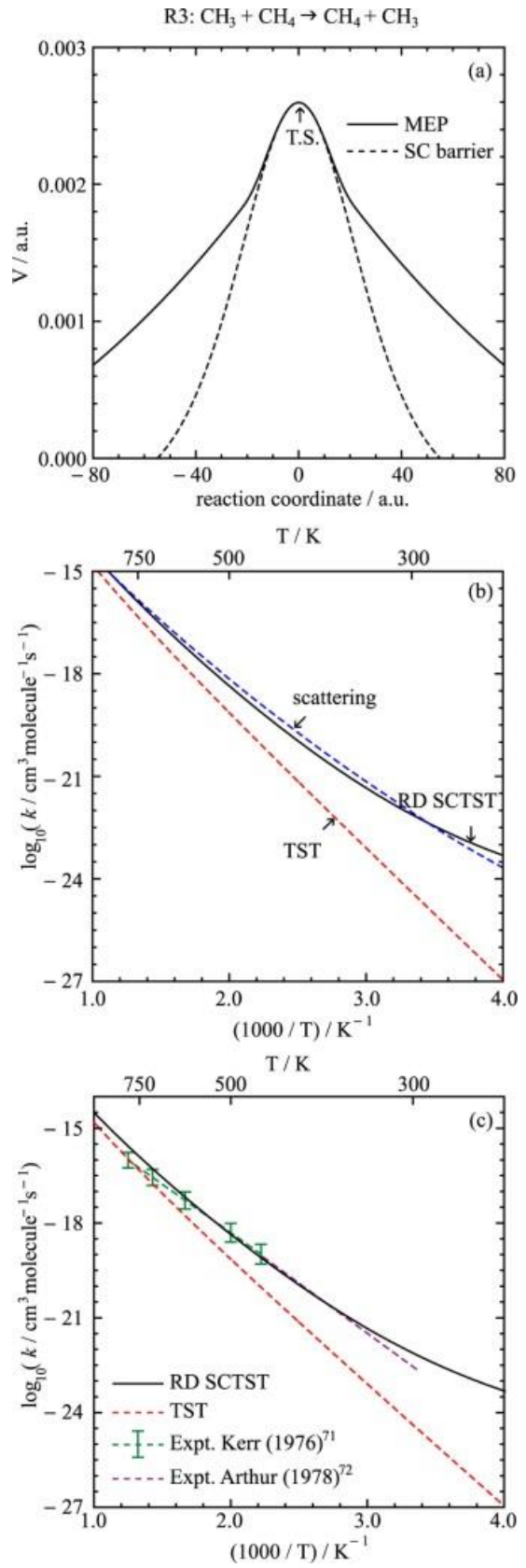

Fig 17. (a) Potential barrier for the $\mathrm{CH}_{3}+\mathrm{CH}_{4} \rightarrow \mathrm{CH}_{4}+\mathrm{CH}_{3}$ reaction along the minimum energy path of the RD PES (solid curve) and the Eckart potential for this system (b) Rate constants for calculated using TST (dashed red line) and RD SCTST ${ }^{116}$ (solid black curve), as well as RDQC scattering rate constants ${ }^{124}$. (c) RD SCTST and TST rate constants compared to experimentally determined rate constants (see Ref 116 for details). 
Also shown in both cases is the effective potential along the minimum energy path for both the QRS potential and the Eckart potential. It can be seen that the effective potential is very "Eckart-like" for the region of the minimum energy path close to the transition state.

Further research on the SCTST has examined the excellent accuracy of a onedimensional 1D-SCTST compared to a full dimensional SCTST for the reactions of $\mathrm{H}$ with $\mathrm{CH}_{4}$ and $\mathrm{C}_{2} \mathrm{H}_{6}{ }^{117,118}$, and for the $\mathrm{CH}_{4}$ and $\mathrm{CH}_{3}$ hydrogen transfer reaction, in which also a hindered rotor modification is applied ${ }^{130}$. The simplest form of the 1D-SCTST only requires 4 single point MP2 calculations in addition to those required for conventional $\mathrm{TST}^{131}$. All the evidence suggests that the 1D-SCTST is a highly computationally inexpensive yet reliable method for predicting rate constants for bimolecular reactions. The theory is also readily applied to polyatomic unimolecular reactions ${ }^{132}$.

Finally, it is often stated, following Wigner ${ }^{8}$, that quantum tunnelling is only important when $\mathrm{H}$ or $\mathrm{D}$ atoms are directly involved in a reaction. The tunnelling probability $P$ for an inverted parabola potential with barrier height $V_{0}$, width $a$, reduced mass $\mathrm{m}$ and collision energy $E$ is ${ }^{133}$

$$
P=\frac{1}{1+\exp \left(2 \pi^{2} a \sqrt{\frac{2 m V_{0}}{h^{2}}}\left(1-\frac{E}{V_{0}}\right)\right)}
$$

Accordingly, a very small barrier width a may compensate for a larger reduced mass $m$ to give appreciable vales of $P$. There is some evidence from physical organic chemistry studies that this may be the case for several special reactions such as the unimolecular rearrangements of methylhydroxycarbene and tert-butylchlorocarbene ${ }^{134}$. Given the progress in extending QRS procedures to organic reactions beyond $\mathrm{H}+\mathrm{CH}_{4}$, this is likely to be a fruitful area for more detailed theoretical and experimental reaction dynamics studies in the future ${ }^{135}$. 


\section{Conclusions}

Since the pioneering Faraday Discussions on Molecular Spectra and Structure in 1929 and Reaction Kinetics in 1937 significant progress has been made in understanding quantum dynamical effects in molecules. Quantum chemistry predictions are now essential to many areas of molecular science as are good calculations on reaction kinetics. The progress in QRS calculations has enabled approximate dynamical theories to be tested for reactions of small molecules so they can then be applied with confidence to larger systems.

These approximate theories can also provide simple pictures of the reaction dynamics which can sometimes be lost in full-dimensional QRS calculations. However, the fulldimensional QRS computations do have the significant advantage that if the potential energy surface being used is highly accurate then they give precise quantitative predictions. Progress in experimental methods has also gone hand-in-hand with that from theory and there is now remarkable and deep understanding of the detailed quantum dynamics of many chemical reactions involving small molecules. We can anticipate further Faraday Discussions on new progress in this field in the years to come.

\section{Acknowledgements}

Permissions for reproduction of figures from the American Institute of Physics, American Chemical Society, Wiley, American Association for the Advancement of Science, Institute of Physics and National Academy of Sciences via the Copyright Clearance Center Rights Link are acknowledged. 


\section{References}

1. Trans. Faraday Soc. 1929, 25, 611.

2. J. E. Lennard-Jones, ref 1, p. 668.

3. R. S. Mulliken, ref 1, p. 634.

4. F. Hund, ref 1, p. 646.

5. V. Henri, ref 1, p. 765.

6. C. V. Raman, ref 1, p. 781.

7. Trans. Faraday Soc. 1938, 34, 1-265.

8. E. Wigner, ref 7, p 29.

9. H Eyring, ref 7, p. 3.

10. H. Eyring, ref 7, p. 41.

11. M. G. Evans, ref 7, p. 49.

12. M. G. Evans and M. Polanyi, ref 7, p. 11.

13. R. P. Bell, ref 7, p. 229.

14. Faraday Discuss. Chem. Soc, 1991, 91.

15. Faraday Discuss. Chem. Soc., 1998, 110.

16. W. H. Miller, ref 14, p1.

17. Faraday Discuss. Chem. Soc, 2017, 195.

18. M. Hochlaf, Phys. Chem. Chem.Phys., 2017, 19, 21236-21261.

19. S. F. Wu, B. R. Johnson and R. D. Levine, Molec. Phys., 1973, 25, 839-856.

20. G. C. Schatz, J. M. Bowman and A. Kupperman, J. Chem. Phys., 1973, 58, 4023-4025.

21. J. T. Muckerman, J. Chem. Phys., 1972, 56, 2997-3006.

22. G. C. Schatz, J. M. Bowman and A. Kupperman, J. Chem. Phys., 1975, 63, 674-685.

23. A. Kuppermann, J. A. Kaye and J. P. Dwyer, Chem. Phys. Lett., 1980, 74, 257-262.

24. G. Hauke, J. Manz and J. Romelt, J. Chem. Phys., 1980, 73, 5040-5044.

25. J. M. Launay and M. Le Dourneuf, J. Phys. B: At. Mol. Phys., 1982, 15, L455-459.

26. G. C. Schatz and A. Kuppermann, J. Chem. Phys., 1973, 59, 964-5.

27. D. C. Clary, J. N. L. Connor and C. J. Edge, Chem. Phys. Lett., 1979, 68, 154-157.

28. J. Manz and J. Romelt, J. Chem. Soc. Faraday Trans., 1990, 86, 1689-1700. 
29. D. C. Clary (ed), The Theory of Chemical Reaction Dynamics, 1986, (NATO ASI Series, Vol 170, Springer).

30. G.C. Schatz and A. Kuppermann , J. Chem. Phys., 1976, 65, 4642-4667.

31. B. Liu and P. Siegbahn, J. Chem. Phys., 1978, 68, 2457-2465.

32. W. H. Miller, Ann. Rev. Phys. Chem., 1990, 41, 245-81.

33. D. E. Manolopoulos and D. C. Clary, Ann. Rep. C. Chem. Soc., 1989, 86, 95-117.

34. S. C. Althorpe and D. C. Clary, Ann. Rev. Phys. Chem., 2003, 54, 493-529.

35. D. H. Zhang and H. Guo, Ann. Rev. Phys. Chem., 2016, 67, 135-158.

36. B. Fu, X. Shan, D. H. Zhang and D. C. Clary, Chem. Soc. Rev., 2017, 24, 7625-7649.

37. R. T Pack and G. A. Parker, J. Chem. Phys., 1987, 87, 3888-3920.

38. D. Skouteris, J. F. Castillo and D. E. Manolopoulos, Comp. Phys. Commun.,2000, 133, 128-135.

39. Z. Sun, H. Guo and D. H. Zhang, J. Chem. Phys., 2010, 132, 084112.

40. J. Chen, Z. Sun and D. H. Zhang, J. Chem. Phys., 2015, 142, 024303.

41. R. T. Skodje, D. Skouteris, D. E. Manolopoulos, S. H. Lee, F. Dong and K. Liu, J. Chem. Phys., 2000, 112, 4536-4552.

42. W. R. Dong, C. L. Xiao, T. Wang, D. X. Dai, X. M. Yang and D. H. Zhang, Science, 2010, $327,1501-2$.

43. M. H. Qiu, Z. F. Ren, L. Che, D. X. Dai, S. A. Harich, X. Y. Wang, X. M. Yang, C. X. Xu, D. Xie, M. Gustafsson, R. T. Skodje, Z. G. Sun and D. H. Zhang, Science, 2006, 311, 1440-1443.

44. Z. F. Ren, L. Che, M. H. Qiu, X. A. Wang, W. R. Dong, D. X. Dai, X. Y. Wang, X. M. Yang, Z. G. Sun, B. Fu, S.Y. Lee, X. Xu and D. H. Zhang, Proc. Natl. Acad. Sci. U. S. A., 2008, 105, 12662-12666.

45.T. Wang, J. Chen, T. G. Yang, C. L. Xiao, Z. G. Sun, L. Huang, D. X. Dai, X. M. Yang and D. H. Zhang, Science, 2013, 342, 1499-1502.

46. D. M. Neumark, Acc. Chem. Res., 1993, 26, 33-40.

47. C. L. Russell and D. E. Manolopoulos, Chem. Phys. Lett., 1996, 256, 465-473. 
48. J. B. Kim, M. L. Weichman, T. F. Sjolander, D. M. Neumark, J. Kłos, M. H. Alexander and D. E. Manolopoulos, Science, 2015, 349, 510-513.

49. H. F. von Horsten and D. C. Clary, Phys. Chem. Chem.Phys., 2011, 13, 4340-4356.

50. T. Westermann, J. B. Kim, M. L. Weichman, C. Hock, T. I. Yacovitch, J. Palma, D. M. Neumark and U. Manthe, Angew. Chem., Int. Ed., 2014, 53, 1122-1126.

51. M. L. Weichman, J. A. Devine, M. C. Babin, J. Li, L. F. Guo, J. Y. Ma, H. Guo and D. M. Neumark, Nature Chem., 2017, 9, 950-955.

52. D. Charlo and D. C. Clary, J. Chem. Phys., 2002, 117, 1660-1672.

53. D. Charlo and D. C. Clary, J. Chem. Phys., 2004, 120, 2700-2707.

54. A. Teplukhin and D. Babikov, Faraday Discuss., 2018.

55. J. C. Polanyi, Angew. Chem. Int. Ed. Engl., 1987, 26, 952-971.

56. Faraday Discuss. Chem. Soc., 1979, 67.

57. D. C. Clary, J. Phys. Chem., 1994, 98, 10678-10688.

58. D. C. Clary, J. Chem. Phys., 1991, 95, 7298-7310.

59. D. C. Clary, J. Chem. Phys., 1991, 95, 3656-3665.

60. D. C. Clary, J. Phys. Chem., 1991, 95, 13664-13669.

61. D. C. Clary, ref. 14, p. 403-405.

62. S. K. Pogrebnya, J. Palma, D. C. Clary and J. Echave, Phys. Chem. Chem. Phys., 2000, 2, 693-700.

63. B. R. Strazisar, C. Lin and H. F. Davis, Science, 2000, 290, 958-961.

64. D. C. Clary, Proc. Nat. Ac. Sci. USA, 2008, 105, 12649-12653.

65. D. H. Zhang, M. A. Collins and S.Y. Lee, Science, 2000, 290, 961-3.

66. C. L. Xiao, X. Xu, S. Liu, T. Wang, W. R. Dong, T. G. Yang, Z. G. Sun, D. X. Dai, X. Xu, D. H. Zhang and X. M. Yang, Science, 2011, 333, 440-442.

67. Z. Zhao, S. Lu and D. H. Zhang, J. Chem. Phys., 2016, 145, 134301.

68. J. Li, B. Jiang and H. Guo, Chem. Sci., 2013, 4, 629-632.

69. H. Song and H. Guo, J. Phys. Chem. A, 2015, 119, 6188-6194.

70. B. Jiang and H. Guo, J. Am. Chem. Soc., 2013, 135, 15251-15256.

71. J. Qi, D. Lu, H. Song, J. Li and M. Yang, J. Chem. Phys., 2017, 146, 124303. 
72. S. Liu, X. Xu and D. H. Zhang, Theor. Chem. Acc., 2012, 131, 1068.

73. G. Nyman and D. C. Clary, J. Chem. Phys., 1994, 101, 5756-5771.

74. B. Kerkeni and D. C. Clary, J. Chem. Phys., 2004, 120, 2308-2318.

75. M. Yang, S.-Y. Lee and D. H. Zhang, J. Chem. Phys., 2007, 126, 064303.

76. S. T. Banks and D. C. Clary, J. Chem. Phys., 2009, 130, 024106.

77. H. F. von Horsten, S. T. Banks and D. C. Clary, J. Chem. Phys., 135, 094311.

78. G. Schiffel and U. Manthe, J. Chem. Phys., 2010, 132, 191101.

79. R. Welsch and U. Manthe, J. Chem. Phys., 2015, 142, 064309

80. R. Ellerbrock and U. Manthe, Faraday Discuss., 2018.

81. D. C. Clary, Ann. Rev. Phys. Chem., 1990, 41, 61-90.

82. D. C. Clary, Molec. Phys., 1984, 53, 3-21.

83. D. C. Clary, Molec. Phys., 1985, 54, 605-618.

84. C. E. Dateo and D. C. Clary, J. Chem. Phys., 1989, 90, 7216-7228

85. D. C. Clary, T. S. Stoecklin and A. G. Wickham, J. Chem. Soc. Faraday Trans., 1993, 89, 2185-2191.

86. D. C. Clary, T. S. Stoecklin and A. G. Wickham, J. Chem. Phys. 1992, 96, 1053-1061.

87. D. C. Clary, J. Chem. Soc. Faraday Trans. 2, 1987, 83, 139-148.

88. T. Stoecklin, D. C. Clary and A. Palma, J. Chem. Soc. Faraday Trans., 1992, 88, 901908.

89. E. Herbst, Int. Rev. Phys. Chem., 2017, 36, 287-331.

90. D. C. Clary, D. Smith and N. G. Adams, Chem. Phys. Lett.,1985, 119, 320-326.

91. I. R. Sims, I. W. M. Smith, D. C. Clary, P. Bocherel and B. R. Rowe, J. Chem. Phys., $1994,101,1748-1751$.

92. A. I. Maergoiz, E. E. Nikitin and J. Troe, Int. J. Mass Spec., 2009, 280, 42-49.

93. A. Kilaj, H. Gao, D. Rösch, U. Rivero, J. Küpper and S. Willitsch, Nature Commun., 2018, 9, 2096.

94. F. A. Gianturco, O. Y. Lakhmanskaya, M. Hernández-Vera, E. Yurtsever and R. Wester, Faraday Discuss., 2018. 
95. D. G. Truhlar, B. C. Garrett and S. J. Klippenstein, J. Phys. Chem., 1996, 100, 1277112800.

96. S. M. Greene, X. Shan and D. C. Clary, J. Chem. Phys., 2016, 144, 244116.

97. C. Qu, Q. Yu and J. M. Bowman, Ann. Rev. Phys. Chem., 2018, 69, 151-175.

98. J. L. Bao and D. G. Truhlar, Chem. Soc. Rev., 2017, 46, 7548-7596.

99. L. Masgrau and D. G. Truhlar, Acc. Chem. Res., 2015, 48, 431-438.

100. I. R. Craig and D. E. Manolopoulos, J. Chem. Phys., 2005, 122, 084106.

101. Q. Meng, J. Chen and D. H. Zhang, J. Chem. Phys., 2015, 143, 101102.

102. Y. V. Suleimanov, F. J. Aoiz and H. Guo, J. Phys. Chem. A., 2016, 120, 8488-8502.

103. W.H. Miller, J. Chem. Phys., 1975, 62, 1899-1906.

104. J. O. Richardson, Ann. Rev. Phys. Chem., 2018, 37, 171-216.

105. A. N. Beyer, J. O. Richardson, P. J. Knowles, J. Rommel and S. C. Althorpe, J. Phys. Chem. Lett., 2016, 7, 4374-4379.

106. G. Laude, D. Calderini, D. P. Tew and J. O. Richardson, Faraday Discuss, 2018.

107. W. H. Miller, J. Chem. Phys., 1975, 62, 1899-1906.

108. W. H. Miller, R. Hernandez, N. C. Handy, D. Jayatilaka and A. Willetts, Chem. Phys. Lett., $1990,172,62-68$.

109. T. L. Nguyen and J. F. Stanton, J. Chem. Phys., 2017, 147, 152704.

110. R. E. Weston, T. L. Nguyen, J. F. Stanton and J. R. Barker, J. Phys. Chem. A, 2013, 117, 821-835.

111. J. R. Barker, T. L. Nguyen and J. F. Stanton, J. Phys. Chem. A, 2012, 116, 6408-6419.

112. T. L. Nguyen, J. F. Stanton and J. R. Barker, J. Phys. Chem. A, 2011, 115, 5118-5126

113. T. L. Nguyen, J. F. Stanton and J. R. Barker, Chem. Phys. Lett., 2010, 499, 9-15

114. T. L. Nguyen and J. F. Stanton, J. Chem. Phys., 2017, 147, 152704.

115. J. F. Stanton, J. Phys. Chem. Lett., 2016, 7, 2708-2713.

116. S. M. Greene, X. Shan and D. C. Clary, J. Phys. Chem. A, 2015, 119, 12015-12027.

117. S. M. Greene, X. Shan and D. C. Clary, J. Chem. Phys., 2016, 144, 084113.

118. S. M. Greene, X. Shan and D. C. Clary, Adv. Chem. Phys., 2018, 163, 117-150.

119. B. Kerkeni and D. C. Clary, Phys. Chem. Chem Phys., 2006, 28, 917-25. 
120. B. Kerkeni and D. C. Clary, J. Chem Phys., 2004, 121, 6809-21.

121. X. Shan and D. C. Clary, Phys. Chem. Chem. Phys., 2013, 15, 18530-18538.

122. X. Shan and D. C. Clary, J. Phys. Chem. A., 2014, 118, 10134-10143.

123. X. Shan and D. C. Clary, Phys. Chem. Chem. Phys., 2013, 15, 1222-1231

124. S. T. Banks, C. S. Tautermann, S. M. Remmert and D. C. Clary, J. Chem. Phys., 2009, 131, 044111.

125. S.T. Banks and D. C. Clary, Phys. Chem. Chem. Phys., 2007, 28, 933-43.

126. S. M. Remmert, S. T. Banks, J. N. Harvey, A. J. Orr-Ewing and D. C. Clary, J. Chem. Phys., 2011, 134, 204311.

127. X. Shan, S. M. Remmert, D. C. Clary, B. Zhang and K. Liu, Chem. Phys. Lett., 2013, 587, 88-92.

128. R. M. Marshall, J. H. Purnell and A. Sheppard, J. Chem. Soc., Faraday Trans. 2, 1986, 82, 929- 935.

129. R. M. Marshall, J. H. Purnell and P. W. Satchell, J. Chem. Soc., Faraday Trans. 2, 1984, 80, 2395- 2403.

130. T. A. H. Burd, X. Shan and D. C. Clary, Chem. Phys. Lett., 2018, 693, 88-94.

131. X. Shan and D. C. Clary, Phil. Trans. Roy. Soc. A, 2018, 376, 20170147.

132 X. Shan, J. C. Vincent, S. Kirkpatrick, M. D. Walker, M. R. Sambrook and D. C. Clary, J. Phys. Chem. A., 2017, 121, 6200-6210.

133. M. D. Harmony, Chem. Soc. Rev. 1972, 1, 211-228.

134. P. R. Schreiner, J. Am. Chem. Soc., 2017, 139, 15276-15283.

135. J. Kästner, Chem. Eur. J., 2013, 19, 8207-8212. 